\title{
Evaluación de compuestos químicos de la variedad Típica de Coffea arabica durante el proceso del tostado artesanal
}

\author{
Evaluation of chemical compounds of the Typica variety of Coffea arabica during the \\ artisan roasting process
}

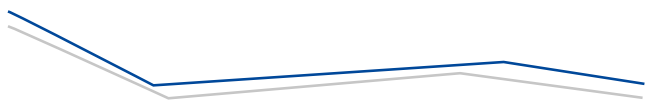

María F. Salazar-Hernández*, Janet A. Lona-Ramírez*, Claudia RiveraDomínguez*, Víctor M. Sánchez-Núñez**, Hugo Rosales-Bravo***, Norma A. Caudillo-Ortega*®

\begin{abstract}
Salazar-Hernández, M. F., Lona-Ramírez, J. A., Rivera-Domínguez, C., SánchezNúñez, V. M., Rosales-Bravo, H., \& Caudillo-Ortega, N. A. (2020). Evaluación de compuestos químicos de la variedad Típica de Coffea arabica durante el proceso del tostado artesanal. Investigación y Ciencia de la Universidad Autónoma de Aguascalientes, 28(81), 32-39.
\end{abstract}

RESUMEN

El café es una bebida y proviene de los granos de café (Coffea arabica), el cual contiene diferentes macromoléculas que durante el tostado sufren una transformación y generan atributos propios del café como sabor, color y otras características físicas. El objetivo es determinar el cumplimiento de los parámetros químicos establecidos en la normatividad vigente en el café arábica con diferentes grados de tueste a partir del tostado artesanal. De acuerdo con los resultados en el presente estudio la composición química cumple con los rangos establecidos en la NMX-

Palabras clave: análisis químico proximal; ART; COffea arabica; CIE Lab; grado de tueste; sacarosa. Keywords: proximal chemical analysis; ART; Coffea arabica; CIE Lab; roasting grade; sucrose.

Recibido: 20 de diciembre de 2019, aceptado: 29 de octubre de 2020

* Ingeniería en Industrias Alimentarias, Instituto Tecnológico Superior de Guanajuato. Carretera Guanajuato a Puentecillas km 10.5, Puentecillas, C. P. 36262, Guanajuato, Gto., México. Correo electrónico: MFSH salazar1025@hotmail.com; janet7154@gmail.com; crivera@itesg.edu. mx; ncaudillo@itesg.edu.mx ORCID: http://orcid.org/0000-0003-27117770; http://orcid.org/0000-0003-1067-8504; http://orcid.org/00000002-5538-4713; http://orcid.org/0000-0002-2895-0919

** Ingeniería Agroindustrial, Universidad Politécnica de Pénjamo. Carretera Irapuato a La Piedad km 44, C. P. 36900, El Derramadero, Guanajuato, Gto., México. Correo electrónico: vmsanchez@uppenjamo.edu.mx ORCID: hHtp://orcid.org/0000-0002-9401-5064

*** Dirección Académica, Colegio de Estudios Superiores Científicos y Tecnológicos del Estado de Guanajuato. Mar de Timor 204, Jardines de la Pradera, C. P. 37536, León, Gto., México. Correo electrónico: hugo. rosalesb@cecyteg.edu.mx ORCID: http://orcid.org/0000-0003-3327$166 \mathrm{X}$

$凶$ Autora para correspondencia
F-013-2000, y el color de cada grado de tueste se ubica en el espacio de color CIE Lab adecuado. Por otro lado, el café con tueste medio (1.146 g/l) presentó mayor contenido en ART, relacionado con las reacciones bioquímicas durante el tostado. Sin embargo, todos los grados de tueste cumplen con los parámetros establecidos.

ABSTRACT

Coffee is a drink and comes from coffee beans (Coffea arabica), which contains different macromolecules that undergo a transformation during roasting, generating attributes typical of coffee such as; flavor, color and other physical characteristics. The objective is to determine compliance with the chemical parameters established in the current regulations in Arabica coffee with different degrees of roasting from artisanal roasting. According to our results, the chemical composition complies with the ranges established in the NMX-F-013-2000, and the color of each degree of roast is located in the appropriate CIE Lab color space. On the other hand, medium roast coffee (1.146 $\mathrm{g} / \mathrm{l})$ presented higher ART content, related to biochemical reactions during roasting. However, all degrees of roast comply with the established parameters.

\section{INTRODUCCIÓN}

El café es un cultivo estratégico en México. A nivel nacional Chiapas es el principal estado productor 
de café con $39 \%$ del volumen nacional, seguido de Veracruz con $30 \%$, mientras que el estado de Oaxaca representa $13 \%$ de la producción de café en México (CEDRASSA, 2018), además es una bebida de gran consumo. En 2018 el informe del mercado de café reportó dos especies que dominan el mercado y la producción: Coffea arabica y Coffea canephora (Robusta).

La etapa del tostado es muy importante en la producción; comprende secado, desarrollo del aroma, descomposición química y tostado completo para adquirir características de color, aroma y sabor (De Luca et al., 2016; Gutiérrez Maydata, 2002). La composición química del grano cambia drásticamente durante el tostado y las altas temperaturas aceleran considerablemente estos cambios (Ajandouz, Tchiakpe, Dalle Ore, Benajiba, \& Puigserver, 2001).

La reacción de Maillard y la caramelización son reacciones bioquímicas presentes en el grano de café verde durante el tostado, en las que intervienen principalmente los azúcares reductores y pueden seguir diversas rutas químicas dependiendo de la composición del grano, produciendo compuestos y cambios físicos que contribuyen a la calidad del café tostado (Cheong et al., 2013; Martins, Jongen, \& Van Boekel, 2001).

El grado de tueste depende del proceso de tostado; dos cafés pueden tener mismas características físicas pero sabores diferentes. El mejor grado de tueste está directamente relacionado con los hábitos de cada país e incluso el paladar de los habitantes de cada zona en cada región. Estas diferencias han dado origen a la preferencia de ciertos grados de tueste basados en el color, sabor y aroma. El grado de tueste se puede clasificar de manera general en 3 tipos: claro, medio y oscuro; a nivel industrial se utilizan tostadoras por cargas y tostadoras en continuo de alto costo que permiten el manejo de variables de tiempo y temperatura mediante sistemas de control automático (Castillo Luzon, Muñoz Ordoñez, \& Engler, 2016). Asimismo, en la calidad del café influye el proceso de tostado, misma que se encuentra regulada por la normatividad mexicana vigente, como la NMX-F-013-2000. Café puro tostado, en grano o molido, sin descafeinar o descafeinado.

La mayoría de los estudios han sido enfocados en la determinación de compuestos del café colombiano (Chaves-Ardila, 2009; Díaz, Ormaza, \& Rojano, 2018) y un estudio reciente caracterizó químicamente el grano verde y tostado de Coffea arabica de dos variedades cosechado en Veracruz, México. El tostado industrial tiene un gran impacto dentro de la industria del café, al tener los parámetros de tiempo y temperatura controlados, de gran impacto en las características organolépticas del café; sin embargo, para productores de pequeña escala está fuera de su alcance.

En el estado de Oaxaca algunos productores con procesos artesanales desarrollan la etapa de tueste para ofertar su producto, de suma importancia ya que en ella se adquieren características que influyen directamente en la calidad. El objetivo de este trabajo fue evaluar compuestos químicos de la variedad Típica de Coffea arabica con diferentes grados de tueste a partir del tostado artesanal y se planteó la hipótesis; los parámetros de algunos compuestos químicos del café con tostado artesanal se mantienen dentro de las especificaciones de la normatividad mexicana vigente.

\section{MATERIALES Y MÉTODOS}

\section{Recolección de la muestra}

Se partió de una muestra de café verde (Coffea arabica) recolectado en la sierra sur de Oaxaca, México. El lote de la muestra fue dividido en submuestras de $100 \mathrm{~g}$ cada una.

\section{Tostado del café}

Se realizó el método artesanal utilizando una cazuela de barro $(26.5 \mathrm{~cm}$ de diámetro, 16.5 de altura y 1 $\mathrm{cm}$ de grosor) y se separaron muestras de café con diferentes grados de tueste correspondientes a claro, medio y oscuro.

\section{Molienda del café}

Los granos de café con diferentes grados de tueste se molieron por separado en un molino manual marca El Rey, después se tamizó en una malla no. 40. 


\section{Análisis químico proximal}

Determinación de humedad. Se utilizó el protocolo descrito en la NMX-F-013- SCFI-200 (Secretaría de Economía, 2000): café puro tostado, en grano o molido, sin descafeinar o descafeinado. Se colocaron $10 \mathrm{~g}$ de muestra molida y tamizada en cajas de aluminio a peso constante. Las cajas con la muestra se colocaron dentro de un horno de convección forzada durante $103^{\circ} \mathrm{C} \pm 1{ }^{\circ} \mathrm{C}$ por $2 \mathrm{~h}$. Posteriormente se calculó el porcentaje de humedad con la siguiente fórmula:

$$
\% \text { humedad }=\frac{M 1-M 2}{M 1-M 0} \times 100
$$

donde $\mathrm{MO}$ es el peso de la caja vacía en g, Ml es el peso de la caja con la muestra húmeda en g y $\mathrm{M} 2$ es el peso de la caja con la muestra seca en $\mathrm{g}$.

Determinación de cenizas. Se utilizó el protocolo descrito en la NMX- F-013-2000 (Secretaría de Economía, 2000): café puro tostado, en grano o molido, sin descafeinar o descafeinado. En un crisol a peso constante se adicionaron $3 \mathrm{~g}$ de la muestra seca, se calcinó en el mechero de Bunsen y los crisoles se colocaron en una mufla a $550^{\circ} \mathrm{C}$ durante 5 h. Los crisoles se enfriaron y el contenido de cenizas se calculó con la siguiente fórmula:

$$
\% \text { cenizas }=\frac{P-p}{M} \times 100
$$

donde $P$ es el peso del crisol con cenizas, $p$ es el peso del crisol vacío, $M$ es el peso de la muestra seca.

Determinación de grasa como extracto etéreo. Esta determinación se realizó de acuerdo al protocolo establecido en la NMX- F-013-2000: café puro tostado, en grano o molido, sin descafeinar o descafeinado. Se pesaron $2 \mathrm{~g}$ de muestra seca y se depositaron en el interior del cartucho de celulosa a peso constante. El cartucho se colocó dentro del extractor Soxhlet, se adicionaron $100 \mathrm{ml}$ de éter de petróleo y se conectó al refrigerante para circulación de agua fría. E calentamiento fue regular y la extracción fue durante $6 \mathrm{~h}$; el éter se evaporó del matraz y se secó a $100^{\circ} \mathrm{C}$ hasta peso constante. Para la expresión de resultados se aplicó la siguiente fórmula:

$$
\% \text { extracto etéreo }=\frac{P f-P 0}{P m} \times 100
$$

Pf es el peso en gramos del matraz con grasa después de la evaporación del solvente. PO es la masa en gramos del matraz sin grasa a peso constante. Pm es el peso en gramos de la muestra seca.

\section{Determinación de fibra cruda en digestión ácida y alcalina.}

\section{Digestión ácida.}

- Se pesan $2 \mathrm{~g}$ de muestra desgrasada (residuo seco del extracto etéreo), se transfieren a un vaso de Berzellius de $600 \mathrm{ml}$. Es importante evitar la contaminación por el papel o el cepillo empleado para retirar la muestra del cartucho.

-Se adicionan $0.5 \mathrm{~g}$ de asbesto, $200 \mathrm{ml}$ de ácido sulfúrico, unas gotas de antiespumante si es necesario.

-Se adapta el vaso a la tapa del condensador de aire y se somete el contenido a ebullición durante 30 min exactamente, rotando periódicamente los vasos para evitar que los sólidos se peguen en el vaso.

-Se filtra al vacío a través del lino sobre un embudo Büchner adaptado al matraz Kitasato.

-Se lava con agua caliente hasta pH neutro.

\section{Digestión alcalina.}

-El residuo se regresa cuantitativamente al vaso Berzellius y se adicionan $200 \mathrm{ml}$ de $\mathrm{NaOH}$ hirviendo.

-Se conecta nuevamente el vaso al condensador y se calienta durante 30 min a ebullición.

-Se filtra nuevamente a través del lino. 
-Se lava hasta la neutralidad y posteriormente con $25 \mathrm{ml}$ de ácido sulfúrico diluido $1.2 \mathrm{M}(1.25 \%)$ caliente (hasta ligera acidez) para eliminar cualquier sustancia que precipite con el álcali y se lava con tres porciones de $50 \mathrm{ml}$ de agua caliente para eliminar el ácido.

-Posteriormente se lava con $25 \mathrm{ml}$ de alcohol etílico y finalmente con éter.

-Se pasa cuantitativamente el residuo a un crisol (a masa constante), se seca a $110^{\circ} \mathrm{C}$ hasta masa constante (aproximadamente $2 \mathrm{~h}$ ), se enfría y se pesa. Se calcina ( $600^{\circ} \mathrm{C}$ durante $30 \mathrm{~min}$ ), se enfría en un desecador y se pesa. La pérdida en masa es la fibra.

La fórmula es la siguiente:

$$
\% \text { fibra cruda }=\frac{P c-P f}{P n} \times 100
$$

donde:

$P c$ es el peso del crisol a peso constante con la muestra seca en g. Pf es el peso del crisol después de la calcinación en g. Pn es el peso de la muestra seca en $\mathrm{g}$.
Determinación del color en café molido con diferentes grados de tueste. Se utilizó un colorímetro (CROMINTEC CHROMATER), tomando como base las coordenadas en el espacio de color CIE L*, a* y b*, para todas las muestras de café molido con diferentes grados de tueste.

Determinación de azúcares reductores totales (ART) en extractos de café molido con diferentes grados de tueste. Para esta determinación se utilizó glucosa como azúcar reductor y se realizó el protocolo descrito por Bello Gil, Carrera Bocourt y Díaz Maqueira (2006) para los extractos de café verde, tueste claro, medio y oscuro.

Análisis estadístico. Para el análisis estadístico se aplicó una comparación de medias con un nivel de significancia de $95 \%, p<0.05$. Se utilizó el paquete estadístico SPSS Statistics versión 25.

\section{RESULTADOS}

\section{Tostado y molienda de los granos de café arábica}

Los granos verdes C. arabica variedad Típica fueron originarios del estado de Oaxaca y mediante un tostado artesanal se obtuvieron tres grados de tueste: claro, medio y oscuro.

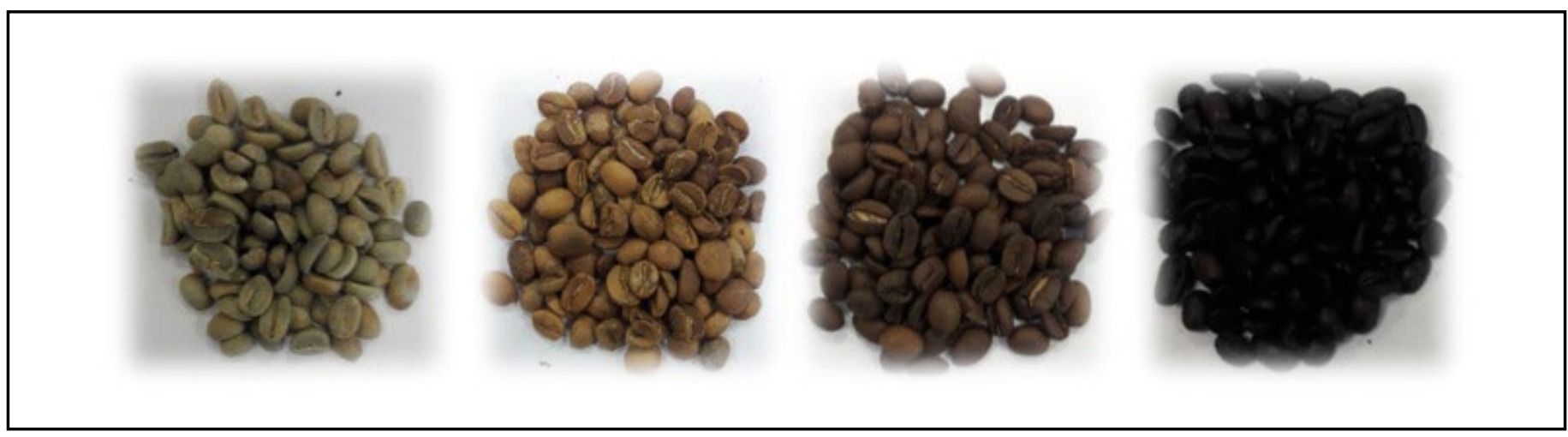

Figura 1. Grados de tueste del C. arabica variedad Típica. De izquierda a derecha: granos de café, granos de café claro, medio y oscuro después del tostado artesanal.

Imagen del equipo de investigación. 
Tabla 1

Composición química de granos de café arábica molido; verde, café con tueste claro, medio y oscuro en base

\begin{tabular}{|c|c|c|c|c|c|}
\hline \multirow[t]{3}{*}{ Componente } & \multicolumn{5}{|c|}{ Grado de tueste del café } \\
\hline & NMX-F- & Verde & Claro| & Medio & Oscuro \\
\hline & $013-2000$ & & & & \\
\hline \multirow[t]{2}{*}{ Humedad } & $6.0 \%$ & $7.35 \pm 0.278$ & $2.47^{*} \pm$ & $2.56^{*} \pm 0.077$ & $2.01^{*} \pm 0.082$ \\
\hline & & & 0.038 & & \\
\hline \multirow[t]{2}{*}{ Cenizas } & $5.0 \% \mathrm{bs}$ & $6.10 \pm 1.744$ & $4.80 \pm 0.195$ & $4.75 \& \& \& \pm$ & $4.56^{*} \pm 0.122$ \\
\hline & & & & 0.046 & \\
\hline \multirow[t]{2}{*}{ Extracto etéreo } & $8.0 \%-$ & $9.73 \pm 1.902$ & $9.01 \& \pm$ & $8.24 \pm 0.140$ & $14.19 * \& \pm \pm$ \\
\hline & $18 \% \mathrm{bs}$ & & 0.870 & & 0.924 \\
\hline \multirow[t]{2}{*}{ Fibra cruda } & -- & $13.79 \pm$ & $13.71 \& \pm$ & $13.43 \& \&$ & $22.07 * \& \& \pm$ \\
\hline & & 1.305 & 2.135 & \pm 2.502 & 0.962 \\
\hline
\end{tabular}

Nota: Los resultados expresan el valor promedio \pm desviación estándar. Diferentes superíndices indican diferencia significativa en los valores promedio de café evaluada a $p<0.05$. *Diferencia significativa; claro, medio, oscuro contra verde. \&Diferencia significativa entre claro y medio. \&\&Diferencia significativa entre claro y oscuro. \&\&\&Diferencia significativa entre medio y oscuro.

Elaboración propia.

\section{Análisis químico proximal}

La tabla 1 muestra el porcentaje de humedad, cenizas, extracto etéreo y fibra cruda del café molido con diferentes grados de tueste.

\section{Determinación de color en el café arábica molido con diferentes grados de tueste}

El color es una característica física de suma importancia, que influye directamente en la calidad del café. Para su valoración se utilizó la escala L, a y b; L representa luminosidad, a y b son coordenadas cromáticas. La tabla 2 muestra los resultados obtenidos en cada uno de los parámetros.

Tabla 2

Parámetros de color CIE del café molido: verde, tueste claro, medio y oscuro

\begin{tabular}{cccc}
\hline Grado de tueste & \multicolumn{3}{c}{ Parámetros de color } \\
\hline & $\mathrm{L}^{*}$ & $\mathrm{a}^{*}$ & $\mathrm{~b}^{*}$ \\
Verde & 13.46 & 21.62 & 21.66 \\
Claro & 29.57 & 26.14 & 29.18 \\
Medio & 16.62 & 16.38 & 16.57 \\
Oscuro & 7.86 & 7.44 & 7.85 \\
\hline
\end{tabular}

Nota: Elaboración propia. 

Determinación de azúcares reductores totales (ART)
en extractos acuosos de café molido

A partir de extracciones acuosas de café molido con diferente grado de tueste se determinaron los ART mediante un método espectrofotométrico. Se obtuvo la curva patrón para cada determinación con un coeficiente de correlación de $R^{2}=0.991$.

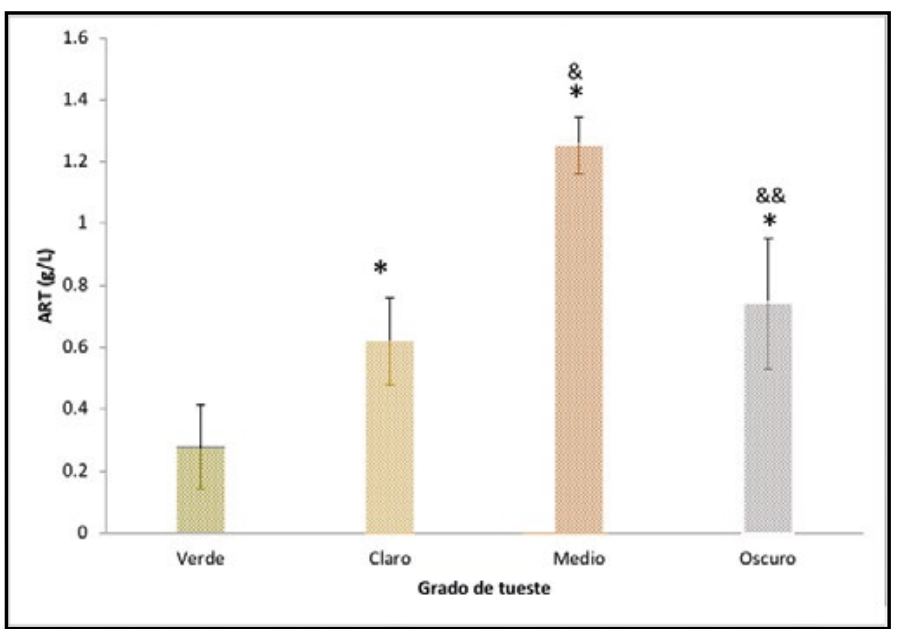

Figura 2. Contenido de ART en extractos acuosos de café con diferente grado de tueste. Los resultados expresan el valor promedio \pm desviación estándar. Diferentes superíndices indican diferencia significativa en los valores promedio de café evaluada a $p<0.05$ *Diferencia significativa; claro, medio, oscuro contra verde. \&Diferencia significativa entre claro y medio. \&\&Diferencia significativa entre claro y oscuro. ${ }^{\& \& \& D i f e r e n c i a ~ s i g n i f i c a t i v a ~ e n t r e ~}$ medio y oscuro.

Elaboración propia.

\section{DISCUSIÓN}

\section{Tostado y molienda de los granos de café arábica}

A nivel mundial el café ha sido estudiado por sus características peculiares. El turco es especial por sus procesos de tostado, molienda, elaboración y almacenamiento; mismos que afectan su calidad y propiedades fisicoquímicas. El tostado en microondas a $350 \mathrm{~W}$ durante 30 min y la molienda con molino cortador son mejores condiciones para obtener las características deseadas en este tipo de café en comparación con los procesos de tostado tradicional en horno o con infrarrojo (Yüksel, Özkara Barut, \& Bayram, 2020).
Los granos verdes C. arabica variedad Típica fueron originarios del estado de Oaxaca. A partir del tostado en cazuela las características físicas del café verde cambiaron conforme transcurría el tiempo adquiriendo tonos específicos, en donde se obtuvieron tres grados de tueste: claro, medio y oscuro (figura 1). Por tanto, el café adquiere las características adecuadas tanto con el tostado artesanal como con el industrial. Posteriormente se realizó la molienda en el tamiz No. 40 y se obtuvieron muestras de café molido fino, de acuerdo con los estándares establecidos en la NMX- F-013-2000 (Secretaría de Economía, 2000).

\section{Análisis químico proximal}

La composición química tiene gran importancia en los alimentos y puede modificarse durante su procesamiento. Los resultados obtenidos muestran el contenido de humedad y cenizas del café con tostado artesanal dentro de las especificaciones que establece la NMX-F-013-2000 (Secretaría de Economía, 2000); al comparar los resultados con el café verde la disminución es significativa. Este comportamiento es esperado, pues parte del agua contenida en los granos de café verde se pierde durante el tostado. En cuanto al contenido de ceniza hay diferencia significativa entre el tueste medio y oscuro; mientras el verde y oscuro presentan mayor y menor contenido, respectivamente; probablemente porque el verde conserva el contenido de celulosa sin modificaciones por el calor.

Por otra parte, el contenido de grasa se encuentra dentro de los parámetros de la normatividad mexicana, siendo el café negro (14.19 \pm 0.924$)$ el de mayor contenido de grasa; además existe una diferencia significativa en el café claro en comparación con el tueste medio y oscuro; estos resultados concuerdan con lo descrito por el grupo de trabajo de Bicho, Leitao, Ramalho y Cebola Lidon (2012) al evaluar granos de café arábica y robusta. Dicho contenido está por debajo de lo reportado por González-Ríos, Suárez-Quiroz, Winkler y Ramírez-Hernández (2018) en café tostado con $16.11 \%$ y $22.16 \%$ en las variedades de $C$. arabica Típica y Milenio, respectivamente. Por tanto, el contenido de grasa aumenta según el grado de tueste.

El contenido de fibra cruda aumentó sorpresivamente en el café oscuro, con diferencia significativa entre los tres grados de tueste, ello concuerda con los parámetros reportados por Puerta-Quintero (2011) para las variedades Caturra y Típica, repre- 
Salazar-Hernández, M. F., Lona-Ramírez, J. A. 38
Rivera-Domínguez, C., Sánchez-Núñez, V. M., Rosales-Bravo, H., \& Caudillo-Ortega, N. A. sentativas de Colombia. Los resultados del análisis químico proximal se encuentran dentro de las especificaciones establecidas por la NMX-F-013-2000 (Secretaría de Economía, 2000), lo que da evidencia de que durante el tostado artesanal la composición química se encuentra dentro de los parámetros para ser comercializado.

\section{Determinación de color en el café arábica molido con diferentes grados de tueste}

Los resultados indican una mayor luminosidad ( $\left.L^{*}\right)$ en el café claro; el café con tueste oscuro tuvo el menor valor en L* y en las coordenadas cromáticas:- $a^{*}$ tiende al color verde y-b* al rojo. Los datos correspondientes a dichas coordenadas en las muestras analizadas colocan al tueste claro dentro del color con tendencia rojo-amarillo, el tueste medio color amarillo-rojo con tendencia a la oscuridad y el tueste oscuro presentó valores más bajos en las coordenadas cromáticas y en luminosidad. Estos parámetros confirman el color negro del café y la reducción durante el tostado.

Los datos del presente estudio concuerdan con trabajos previos, donde se observó que la L* de las muestras de café disminuyó con el aumento del tiempo de tostado (Díaz et al., 2018). El cambio de coloración de verde-pardas-negras se genera por las melanoidinas durante las reacciones de caramelización, Maillard y polimerización (Lee, Cheong, Curran, Yu, \& Liu, 2015; Moreira et al., 2017); además contribuyen a la formación del olor.

\section{Determinación de azúcares reductores totales (ART) en extractos acuosos de café molido}

El contenido de azúcares reductores en los extractos acuosos aumentó en el tueste claro y medio, con características de aroma agradables, siendo el medio el que presentó un olor característico al café comercial y dentro del $5.5 \%$ que establece la NMX-F-013-2000 (Secretaría de Economía, 2000). Los tres tuestes tienen diferencia significativa en comparación con el café verde (figura 2). Asimismo, se presentó una diferencia significativa al comparar los tuestes claro vs. medio y claro vs. Oscuro. Los ART se establecen en base seca de 0.1 y $0.3 \%$ para café verde y tostado, respectivamente (Puerta-Quintero, 2011).

CONCLUSIONES

Con el análisis químico proximal del café con tueste artesanal se verificó el cumplimiento de los parámetros determinados dentro de las especificaciones establecidas en la NMX-F-013-2000 (Secretaría de Economía, 2000). El contenido de azúcares presente en el café verde cambia respecto a la temperatura y tiempo, siendo el de tueste medio el de mayor contenido de ART.

El de tueste oscuro presentó menor contenido en ART, posiblemente por la degradación de los azúcares y formación de compuestos que potencian el sabor amargo. El color es fundamental en el café y es característico de cada grado de tueste, los resultados avalaron las coordenadas en el espacio de color correspondiente a cada grado de tueste.

A partir de estos resultados los pequeños productores de café pueden realizar la etapa de tostado de forma artesanal. Como perspectiva se puede realizar una carta de color para un método comparativo para productores a pequeña escala con el que puedan obtener un tueste en rango correcto.

\section{Agradecimientos}

Los autores desean agradecer al Tecnológico Nacional de México por su financiamiento. Por su dedicación y compromiso a estudiantes del servicio social Sandra Paola Lona Luna, Luis Enrique Blancas Hernández y Arelí Guadalupe Salas Amézquita; así como a la Universidad Politécnica de Pénjamo (UPP) en el estado de Guanajuato. 
- $\quad$ Ajandouz, E. H., Tchiakpe, L. S., Dalle Ore, F., Benajiba, A., \& Puigserver, A. (2001). Effects of pH on caramelization and maillard reaction kinetics in fructose-lysine model systems. Journal of Food Science, 66 (7), 926-931.

- Bello Gil, D., Carrera Bocourt, E., \& Díaz Maqueira, Y. (2006). Determinación de azúcares reductores totales en jugos mezclados de caña de azúcar utilizando el método del ácido 3,5 dinitrosalicílico. ICIDCA. Sobre los Derivados de la Caña de Azúcar, 40(2), 45-50.

- Bicho, N. C., Leitao, A. E., Ramalho, J. C., \& Cebola Lidon, F. (2012). Use of colour parameters for roasted coffee assessment. Food Science and Technology, 32 (3), 436-442.

- Castillo Luzon, M. A., Muñoz Ordoñez, M., \& Engler, F. (2016). Manual básico de buenas prácticas para el tostado del café (pp. 7-25). Ecuador: Ministerio de Industrias y Productividad. Recuperado de https://www.redalyc.org/articulo. oa?id=2231 20664006

- Chaves-Ardila, L. G. (2009). El café tostado y molido: Caracterización de la industria torrefactora nacional. Umbral Científico, 14, 98-106. Recuperado de https://www.redalyc. org/pdf/304/30415059009.pdf

- Centro de Estudios para el Desarrollo Rural Sustentable y la Soberanía Alimentaria \& Cámara de Diputados LXIII Legislatura. (2018). Reporte: El café en México. Diagnóstico y perspectiva. México: Autor.

- Cheong, M. W., Tong, K. H., Ming-Ong, J. J., Liu, S. Q., Curran, P., \& YU, B. (2013). Volatile composition and antioxidant capacity of Arabica coffee. Food Research International, $51(1), 388-396$.

- De Luca, S., De Filippis, M., Bucci, R., Magri, A. D., Magri, A. L., \& Marini, F. (2016). Characterization of the effects of different roasting conditions on coffee samples of different geographical origins by HPLC-DAD, NIR and chemometrics. Microchemichal Journal, 129, 348-361.

- Díaz, F. O., Ormaza, A. M., \& Rojano, B. A. (2018). Efecto de la tostión del café (Coffea arabica L. var. Castillo) sobre el perfil de taza, contenido de compuestos antioxidantes y la actividad antioxidante. Información Tecnológica, 29(4), 31 42.

- González-Ríos, O., Suárez-Quiroz, M. L., Winkler, R., \& RamírezHernández, A. K. (2018). Caracterización química en grano verde y tostado de una variedad de Coffea arabica L. cosechado en 2016 en Huatusco, Veracruz México. Revista
Colombiana de Investigaciones Agroindustriales, 5(2) 86-97. doi: 10.23850/24220582.1596

- Gutiérrez Maydata, A. (2002). Café, antioxidantes y protección a la salud. MEDISAN, 6(4), 72-81. Recuperado de https://www.imbiomed.com.mx/ articulo.php?id=13580

- $\quad$ Lee, L. W., Cheong, M. W., Curran, P., Yu, B., \& Liu, S. Q. (2015). Coffee fermentation and flavor - An intricate and delicate relationship. Food Chemistry, 185, 182191. doi: 10.1016/j.foodchem.2015.03.124

- Martins, S. I. F. S., Jongen, W. M. F., \& Van Boekel, M A. J. S. (2001). A review of Maillard reaction in food and implications to kinetic modelling. Trends in Food Science \& Technology, 11 (9-10), 364-373.

- Moreira, A. S. P., Nunes, F. M., Simões, C., Maciel, E., Domingues, P., Domingues, M. R. M., \& Coimbra, M. A. (2017). Transglycosylation reactions, a main mechanism of phenolics incorporation in coffee melanoidins: Inhibition by Maillard reaction. Food Chemistry, 227, 422-431.

- Puerta-Quintero, G. I. (2011). Composición química de una taza de café. Avances Técnicos Cenicafé, $414,1-12$

- Secretaría de Economía. (2000). Norma Mexicana NMX-F-013-2000. Café puro tostado, en grano o molido, sin descafeinar 0 descafeinado. Especificaciones y métodos de prueba. Recuperada de https://www.colpos.mx/bancodenormas/ nmexicanas/NMX-F-013-2000.PDF

- $\quad$ Yüksel, A. N., Özkara Barut, K. T., \& Bayram, M. (2020). The effects of roasting, milling, brewing and storage processes on the physicochemical properties of Turkish coffee. LWT, 131, 109711. doi: 10.1016/j. Iwt.2020.109711 